\title{
A Systematic Review of Health Economic Evaluation on Targeted Therapies for First-Line Treatment of Metastatic Non-Small Cell Lung Cancer (NSCLC): Quality Evaluation
}

This article was published in the following Dove Press journal:

Cancer Management and Research

Jie Zhaol,2
Shuzhang Du
Yumei Zhu'
Yan Liang
Jingli Lu²
Feng Chang (D)

'School of International Pharmaceutical Business, China Pharmaceutical University, Nanjing, People's Republic of China;

${ }^{2}$ Department of Pharmacy, The First Affiliated Hospital of Zhengzhou University, Zhengzhou, People's Republic of China
Correspondence: Feng Chang School of International Pharmaceutical Business, China Pharmaceutical University, \#639 Longmian Avenue, Jiangning District, Nanjing 2III 98, People's Republic of China Email cpucf1910@I26.com
Background: Evolving practices in non-small cell lung cancer (NSCLC) therapy inevitably affect health care budgets, especially through the introduction of targeted therapies. This results in a rise of health economic evaluations (HEEs) in this domain. This article reviews the quality of the economic evidence of targeted therapies used in metastatic NSCLC.

Methods: A literature search was conducted using PubMed, Cochrane, Embase and CRD (University of York Centre for Reviews and Dissemination) databases to identify topical original articles published between $1 / 1 / 2000$ and 3/31/2019. A quality of reporting assessment using the CHEERS (Consolidated Health Economic Evaluation Reporting Standards statement) checklist was converted into a quantitative score and compared with the results of a QHES (Quality of Health Economic Studies) evaluation. Components of QHES were also used to analyze the validity of primary outcomes, consideration of heterogeneity and rationality of main assumptions of models in modeling studies.

Results: In total, 25 HEEs were obtained and analyzed. From the CHEERS assessment, it was found that method description integrity (including setting, perspective, time horizon and discount rate), justification of data sources and a heterogeneity description were often absent or incomplete. Only five examined studies met the accepted standard of good quality. Modeled articles were examined with the QHES instrument, and a lack of illustrated structure, population variability, formula of the transitioning probability and justification for the choice of the model were the most frequently observed problems in the selected studies. After quantification, the CHEERS scores and QHES scores did not differ significantly.

Conclusion: Current NSCLC models generally lack consideration for demographic heterogeneity and transparency of data description, and it would be difficult to transfer or generalize from the scientific literature to real-world evidence-based decision-making. Frameworks of future models should be informed and justified based on the validity of model results and the improvement of modeling accuracy.

Keywords: quality evaluation, targeted therapies for NSCLC, CHEERS, QHES

\section{Introduction}

Lung cancer is the most common form of cancer and the leading cause of cancerrelated mortality worldwide, resulting in about 1.7 million deaths each year. ${ }^{1}$ Nonsmall-cell lung cancer (NSCLC) accounts for approximately 85-90\% of all lung cancer cases, ${ }^{2}$ inherently having a noticeable impact on health-care budgets. Among those cases, $10-20 \%$ of white patients and about $48 \%$ of Asian patients carry 
mutations in the epidermal growth factor receptor (EGFR) that play a key role in carcinogenesis. ${ }^{3}$ In the clinical therapy for advanced NSCLC, the past two decades brought an important change: the use of targeted anticancer drugs was established in the context of inoperable treatment. Since the landmark I-PASS trial established the role of first-generation EGFR-tyrosine kinase inhibitors (TKIs) as the preferred first-line therapy for EGFR mutant tumors, gefitinib, erlotinib and afatinib (second-generation EGFR-TKI) gained global approval for this clinical treatment. $^{4}$ More recently, osimertinib was developed as a third-generation drug for NSCLC with EGFR T790M resistance mutations. ${ }^{5}$

Ample evidence shows that the cost of cancer therapy is becoming unaffordable in many countries. ${ }^{6}$ Considering the limited nature of healthcare resources, systematic analyses of various medical projects can help to identify relevant options. The methodology applied to analyze the inputs and outputs of medical activities, as well as to make explicit whether a new intervention or strategy is worthwhile economically, can be defined as health economic evaluation (HEE). It is accordingly so that the general interest in HEE increases each year. The identification of various costs and measurement in monetary units in most HEEs is similar, but the nature of the results of various HEEs may be quite different depending on the different techniques used. In short, costeffectiveness analysis (CEA) relates a change in costs to the difference in health effects, expressed in natural units such as life-years gained (LYG); whereas a cost-utility analysis (CUA) expresses the health effect as quality adjusted life years (QALYs) gained.

Transparency of reporting is an essential factor needed to evaluate methods, assumptions, models and possible biases of HEE results. To address this question, many instruments were developed to evaluate the methodological quality of health economics research. The "British Medical Journal", the "Drummond" and the "CHEC" checklists are wellknown instruments used for qualitative evaluation. ${ }^{7-9}$ When the International Society for Pharmacoeconomics and Outcomes Research (ISPOR) issued the Consolidated Health Economic Evaluation Reporting Standards statement (CHEERS), the objective was to guide and further standardize the reporting of economic evaluations. ${ }^{10}$ In contrast, the Quality of Health Economic Studies (QHES) is an instrument intended and validated for quantitative scoring. ${ }^{11}$

In the present study, qualitative and quantitative scoring assessments were performed on HEE publications focusing on non-small-cell lung cancer after a systematic literature review. The quality of reporting of the selected studies was appraised using the CHEERS checklist, and the results were applied as a scoring system compared with the QHES evaluation. This article reports the results of evaluation and comparison using both instruments, and describes remaining shortcomings and methodological questions in the available literature of HEEs in targeted therapy for NSCLC.

\section{Materials and Methods Search Strategies and Study Selection}

Search strategies were designed for accessing PubMed, the Cochrane Library, EMBASE and the University of York Centre for Reviews and Dissemination (CRD) Database, encompassing literature published between 1/1/2000 and 3/ $31 / 2019$ to encompass the novel targeted therapies that emerged this century. The searches were restricted to publications written in English or Chinese due to the linguistic capabilities of the authors of this analysis. A detailed description of the included index terms and free-text words can be found in Supplementary file 1.

Table 1 describes the study selection criteria. After removal of duplicates, titles and abstracts were screened based on presence of both economic aspects and treatment of NSCLC. Final selection required comparison of different analytical strategies in NSCLC, specifying the targeted drug cost as the real result of a costing exercise. Only original studies published in available full text were included. Conference articles, reviews and position papers were excluded. The eligibility of the studies for review was assessed subjectively, and uncertainties were resolved in discussion amongst the coauthors of the review.

\section{Quality Evaluation}

The CHEERS statement contains a 24-item checklist, that is used to optimize and improve reporting quality of health economic evaluations. ${ }^{10}$ All of the 24 items were examined in each article by two review authors independently (ZJ and LY). In cases of disagreement, a consensus was reached through subjective discussion. Furthermore, in order to compare the results from CHEERS with the qualitative QHES evaluation, the CHEERS checklist was converted into a quantitative score. Since each item focuses on one single aspect, equal weights were allocated, with a score of ' 1 ' if complete, ' 0.5 ' for partial report and ' 0 ' for not mentioned. This method is generally in agreement with the respective scores of 2, 1 and 0 as proposed by Monten et al, ${ }^{12}$ who also assigned an equal weight to all items. 
Table I Paper Selection Criteria

\begin{tabular}{|l|l|}
\hline Population & $\begin{array}{l}\text { Studies of Participants Diagnosed with NSCLC, } \\
\text { Restricted Based on the First-Line Treatment. }\end{array}$ \\
\hline $\begin{array}{l}\text { Intervention/ } \\
\text { Comparison }\end{array}$ & $\begin{array}{l}\text { Studies about treatments with specific targeted } \\
\text { agents: afatinib, gefitinib, erlotinib and } \\
\text { osimertinib }\end{array}$ \\
\hline Outcomes & Costs \\
\cline { 2 - 3 } & $\begin{array}{l}\text { Clinically relevant outcome measures (QALY or } \\
\text { Life year gained) }\end{array}$ \\
\hline Study design & $\begin{array}{l}\text { Economic evaluations (cost comparison, cost } \\
\text { effectiveness, cost utility), health technology } \\
\text { assessments }\end{array}$ \\
\hline
\end{tabular}

The QHES instrument is a dichotomous scoring system that was designed to evaluate three common types of health economic analyses: cost-minimization (CMA), costeffectiveness (CEA), and cost-utility (CUA). ${ }^{13}$ Each published study was scored in 16 items, allocating a Yes (fulfilled) or No (not fulfilled) per item, and each score was multiplied by a set weight, varying between 1 and 9 , to obtain a total score out of 100 points possible. ${ }^{14}$ No partial points per item are intended or allowed in this analysis. ${ }^{13}$ The QHES evaluation was conducted independently by the same two researchers to address and overcome interpretational problems.

Resulting scores of the two instruments used for each published study were converted into percentages to allow for direct comparison.

\section{Statistical Analysis}

Instruments were compared using the paired Wilcoxon rank test for continuous variables. The $\mathrm{R}$ statistical environment (version 3.5.3, TUNA Team, Tsinghua University, China) was used to develop and solve the comparison. A p-value of $<0.05$ was considered as the threshold for statistical significance.

\section{Results}

The database search identified 506 publications, yielding 366 after removal of duplicates and 188 after screening titles and abstracts. Finally, 25 full-text articles were identified that fulfilled the inclusion criteria. The CONSORT diagram is illustrated in Figure 1.

\section{Study Characteristics}

Detailed characteristics of the included 25 publications are summarized in Table 2. All selected studies represented a full economic evaluation, examining both costs and effectiveness (CEA) or utilities (CUA). Out of the 25 publications, 22 took variable estimates in the analysis from randomized-controlled trials (RCTs), mostly derived from the LUX-Lung 3.6 and 7, OPTIMAL, EURTAC, SATURN, GFPC, BR.21 and FLAURA trials. The other three publications were based on hospital medical records. Nine studies specifically demonstrated the patient population, and the study sample sizes varied from 41 to a cohort of 731 patients.

The majority of articles compared targeted therapies to standard chemotherapy $(\mathrm{n}=16)$, being afatinib versus chemotherapy $(\mathrm{n}=2)$, erlotinib versus chemotherapy/placebo/ best supportive care (BSC) $(n=12)$ and gefitinib versus chemotherapy/routine care $(n=2)$. Twelve articles evaluated the cost-effectiveness of treatments between the four available first-line strategies among NSCLC patients harboring EGFR mutations. Four of these articles compared afatinib to gefitinib in three countries, two studies estimated the different economic impact between afatinib and erlotinib in two countries, another two publications addressed the efficacy and cost-effectiveness of erlotinib versus gefitinib in one country, and four articles compared the cost-effectiveness between osimertinib and other three first/second-generation EGFR-TKIs in four countries.

In structure, 14 articles developed a Markov model to compare the cost-effectiveness of first-line targeted therapies and chemotherapy. The majority of these created three similar health states, except for one study ${ }^{15}$ that developed seven health states to compare the short-term impact of maintenance therapy. Only two studies were based on decision trees without a Markov component ${ }^{16,17}$ and four were adopted from the same partitioned survival model with three health states. ${ }^{18-21}$ Another five studies ${ }^{22-26}$ were found that reported no economic model.

\section{Quality of Reporting}

The results of the assessment of reporting quality for each study are summarized in Supplementary file 2, and items of CHEERS and QHES questionnaires were listed in Supplementary file 3. Figure 2 shows a representation of the fulfillment of the CHEERS criteria and a sorting of completeness of the items. The score on the 24-item CHEERS checklist ranged from 12 to 21 among the selected studies, and the average score was 17.84. According to the previously accepted descriptors reported by Hong et al, ${ }^{27}$ the publications were categorized as being of good reporting quality if they were scored 20-24, and were deemed to be 


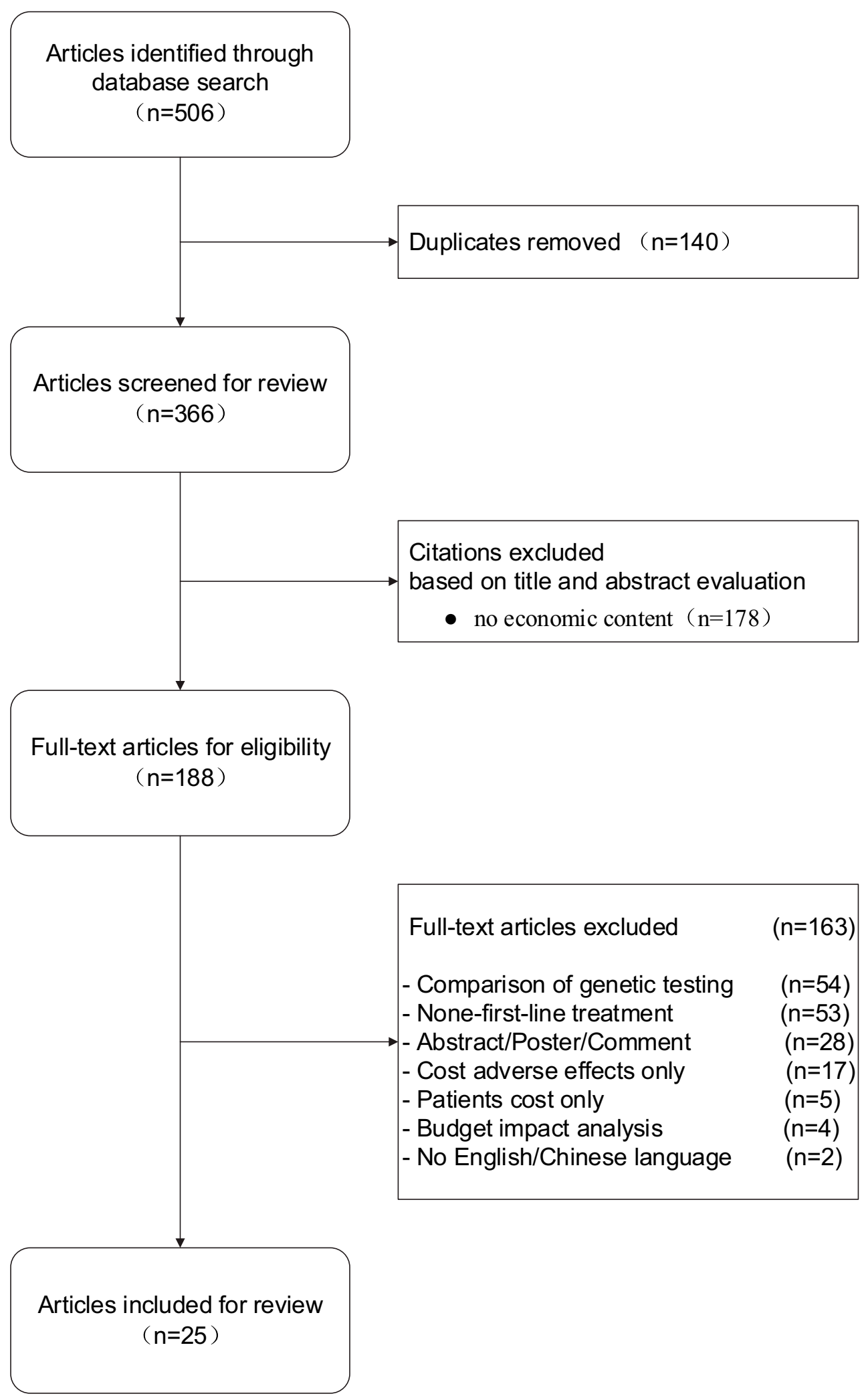

Figure I Consort diagram

of moderate and low reporting quality if they were scored 14-19.5 and $<14$, respectively. Only five studies were of good quality based on the CHEERS checklist score, while
18 were of moderate quality and two were of low quality. The quality rankings of these studies were not correlated in relation with the years of publication. The treatments evaluated 


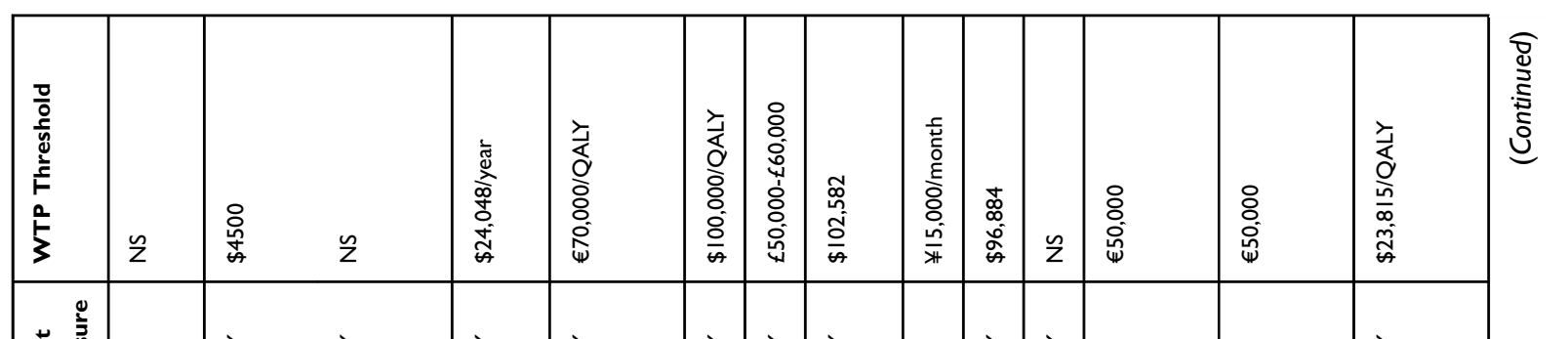

\begin{tabular}{|c|c|c|c|c|c|c|c|c|c|c|c|c|c|c|}
\hline 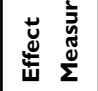 & $\frac{\mathfrak{n}}{\Sigma}$ & ગ્ & 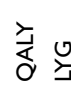 & $\frac{\grave{\partial}}{\partial}$ & $\frac{\grave{\partial}}{\partial}$ & 文 & ટે & ટ્ટે & $\underline{\underline{\alpha}}$ & 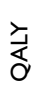 & ટૅ & У્ & $\stackrel{\searrow}{\beth}$ & ป઼ \\
\hline 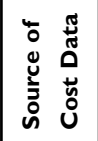 & 出 & $\tilde{z}$ & 出 & $\tilde{\digamma}$ & 岀 & 出 & 出 & $\tilde{\vdash}$ & $\tilde{\vdash}$ & $\tilde{r}$ & $\tilde{r}$ & $\tilde{\llcorner}$ & $\tilde{\vdash}$ & $\tilde{\vdash}$ \\
\hline
\end{tabular}

\begin{tabular}{|c|c|c|c|c|c|c|c|c|c|c|c|c|c|c|}
\hline 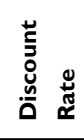 & । & $\stackrel{\circ}{~}$ & $\stackrel{\circ}{\text { ले }}$ & 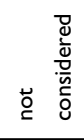 & ○े & $\stackrel{\circ}{\text { ले }}$ & 1 & $\stackrel{\circ}{\text { ले }}$ & $\tilde{z}$ & ले & $\tilde{z}$ & $\stackrel{\text { ஸें }}{\text { ஸे }}$ & ஸें & ํํ \\
\hline 总 & ๕气 & 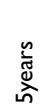 & 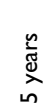 & 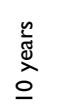 & 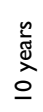 & 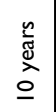 & 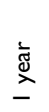 & $\check{z}$ & $\check{z}$ & $\begin{array}{l}\text { 駡 } \\
\text { 人̃ } \\
\underline{0}\end{array}$ & $\check{z}$ & 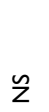 & $\check{z}$ & 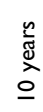 \\
\hline
\end{tabular}

\begin{tabular}{|c|c|c|c|c|c|c|c|c|c|c|c|c|c|c|}
\hline 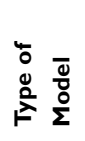 & $\stackrel{\mathrm{Z}}{\mathrm{z}}$ & 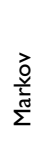 & 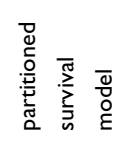 & 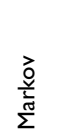 & 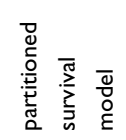 & $\frac{\vec{े}}{\frac{i}{\bar{m}}}$ & $\tilde{z}$ & 旁 & 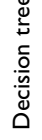 & $\frac{\vec{c}}{\frac{\vec{q}}{\bar{c}}}$ & $\tilde{z}$ & 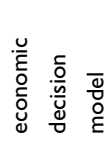 & 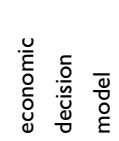 & 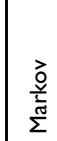 \\
\hline 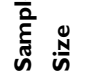 & $\bar{\gamma}$ & $\stackrel{\stackrel{m}{m}}{ }$ & $\tilde{z}$ & $\underset{m}{\infty}$ & $\frac{\sigma}{m}$ & $\tilde{z}$ & ?̊ & $\tilde{z}$ & $\stackrel{\circ}{\circ}$ & $\tilde{z}$ & 으 & $\check{z}$ & $\tilde{z}$ & $\tilde{z}$ \\
\hline
\end{tabular}

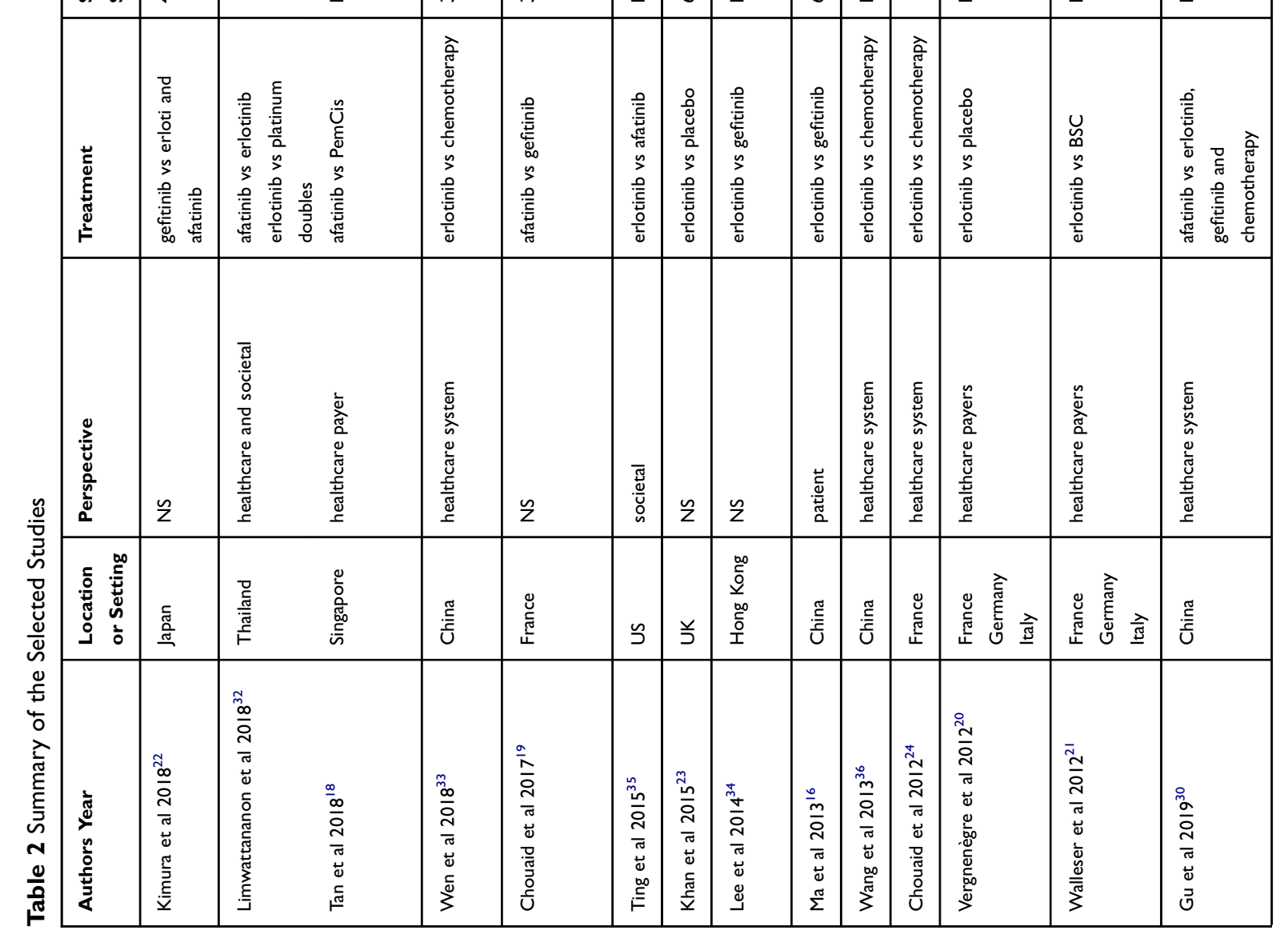




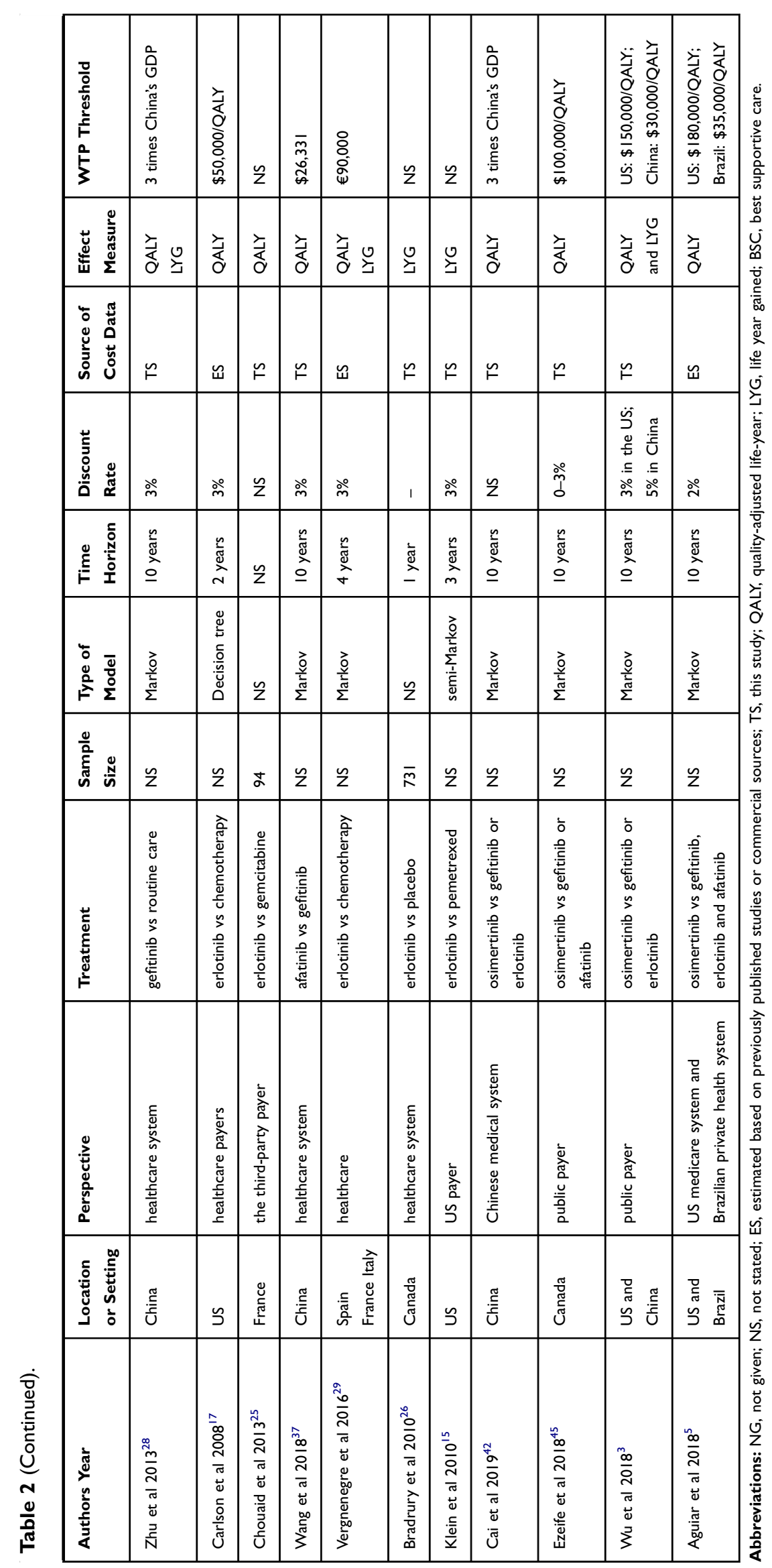




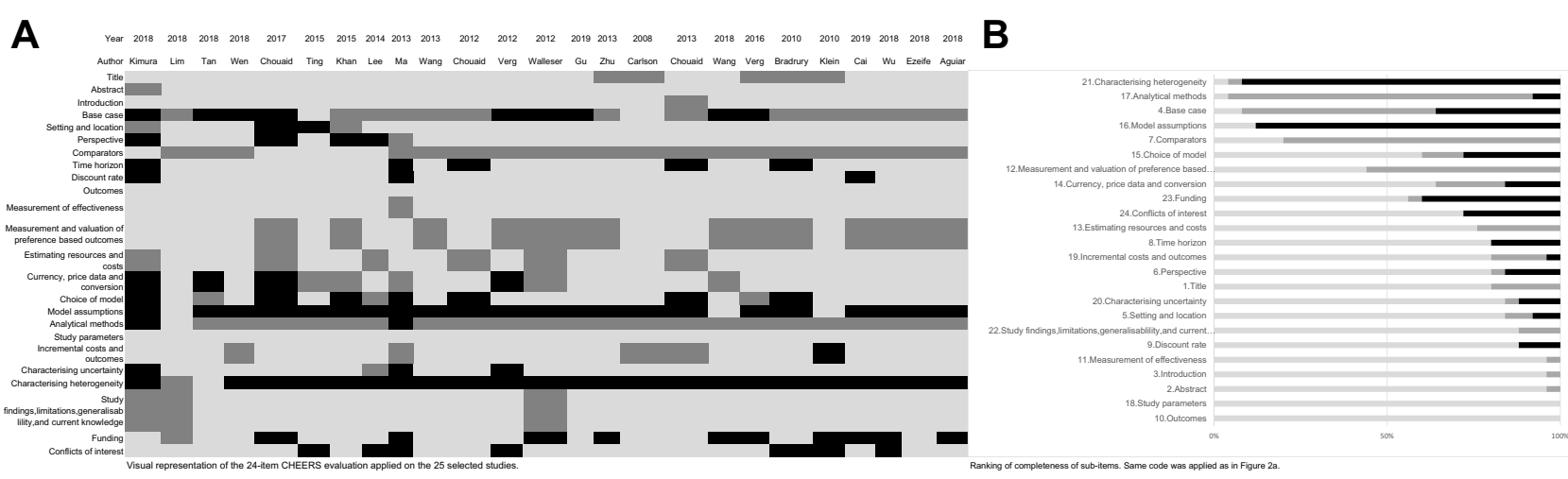

Figure 2 Overview of evaluation using CHEERS criteria, per article (left) and per item (right). (A) (left): Visual representation of the 24-item CHEERS evaluation applied on the 25 selected studies. (B) (right): Ranking of completeness of sub-items. Same code was applied as in Figure 2A.

were typically described in the title of the publication, although five papers did not use the title to describe the interventions they compared. ${ }^{15,17,26,28,29}$ In addition, the setting, perspective, time horizon and discount rates were not always included; results of uncertainty analyses, choice for health outcomes, findings and conflicts of interest were also not provided in all articles. For measurement and valuation of preference-based outcomes, only two papers were based on a systematic review, ${ }^{22,30}$ while the others referred to the source of utilities chosen without justifying the selection.

The results of the quality assessment of each article using the QHES instrument are presented in Supplementary file 2, which shows how often each criterion was met by each of the 25 studies. According to Spiegel et al, ${ }^{31}$ studies can be grouped by the following quartiles: (1) extremely poor quality (0-24); (2) poor quality (25-49); (3) fair quality (50-74); and (4) high quality (75-100). Less than half (48\%) of publications reviewed were thus classified as high quality, and three studies $(14.3 \%)$ are of poor quality, ${ }^{22,24,25}$ while the average score of the selected studies was 71.52. The source of utility values that all selected studies extracted were also evaluated, and 17 out of 25 papers used values obtained from previously published literature, five studies derived utility data from EQ-5D or another quality of life survey, ${ }^{17-19,23,32}$ and three studies used survival data from a single medical institution $^{16}$ or clinical trials. ${ }^{20,21}$ A pairwise comparison demonstrated that there was no statistically significant difference between the results of the CHEERS and QHES instruments, $\mathrm{p}=0.51$, illustrated in Figure 3 .

Although there was no major difference observed based on the type of economic analysis, there were some differences noted in the ratings of articles with diverse models. As for the five model-free publications, four of them scored lower in QHES than CHEERS, ${ }^{22-25}$ as two items in QHES have a stated demand for an economic model. According to the Markov model articles, for item 3 in QHES, which weighted the subgroup analysis the lowest scoring, and items 16 and 21 in CHEERS, requiring specific model assumptions and descriptions of heterogeneity, there are four publications with large score difference. ${ }^{5,15,33,34}$ Although the same-partitioned survival model was undertaken by four articles, ${ }^{18-21}$ no justification for the choice of the model was disclosed in two of them, ${ }^{20,21}$ which resulted in the inconsistency of scores evaluated by the CHEERS and QHES instruments.

\section{Modeling Articles}

For the articles that used the Markov model, there was no illustrated structure of the model in three studies, ${ }^{29,34,35}$ and three others did show the Markov model tree. ${ }^{15,33,36}$ Two studies $^{33,37}$ manifested the formula of the transitioning probabilities specifically from each stage to the next, while most others demonstrated the rates or the probability that were calculated from clinical trials. No justification for the choice of the model was given by six of the articles. ${ }^{16,20,21,28,29,32}$ Most of the studies stated the effectiveness value in outcomes as QALY or LYG, and one article ${ }^{22}$ used median survival time (MST) to evaluate the therapeutic effect of the regimens. As most studies used data from RCTs, characterization of heterogeneity was generally not provided. Only Wang et $\mathrm{al}^{37}$ reported explicitly by variations between subgroups of patients with different genotype baselines. Although some studies described the base case population, most did not present characteristics or the reasons they were chosen. Among the Markov models that simulated transition of individuals across NSCLC health states, most were based on the same concept of clinical condition or symptom, but the study methods, the components of the numerator and 


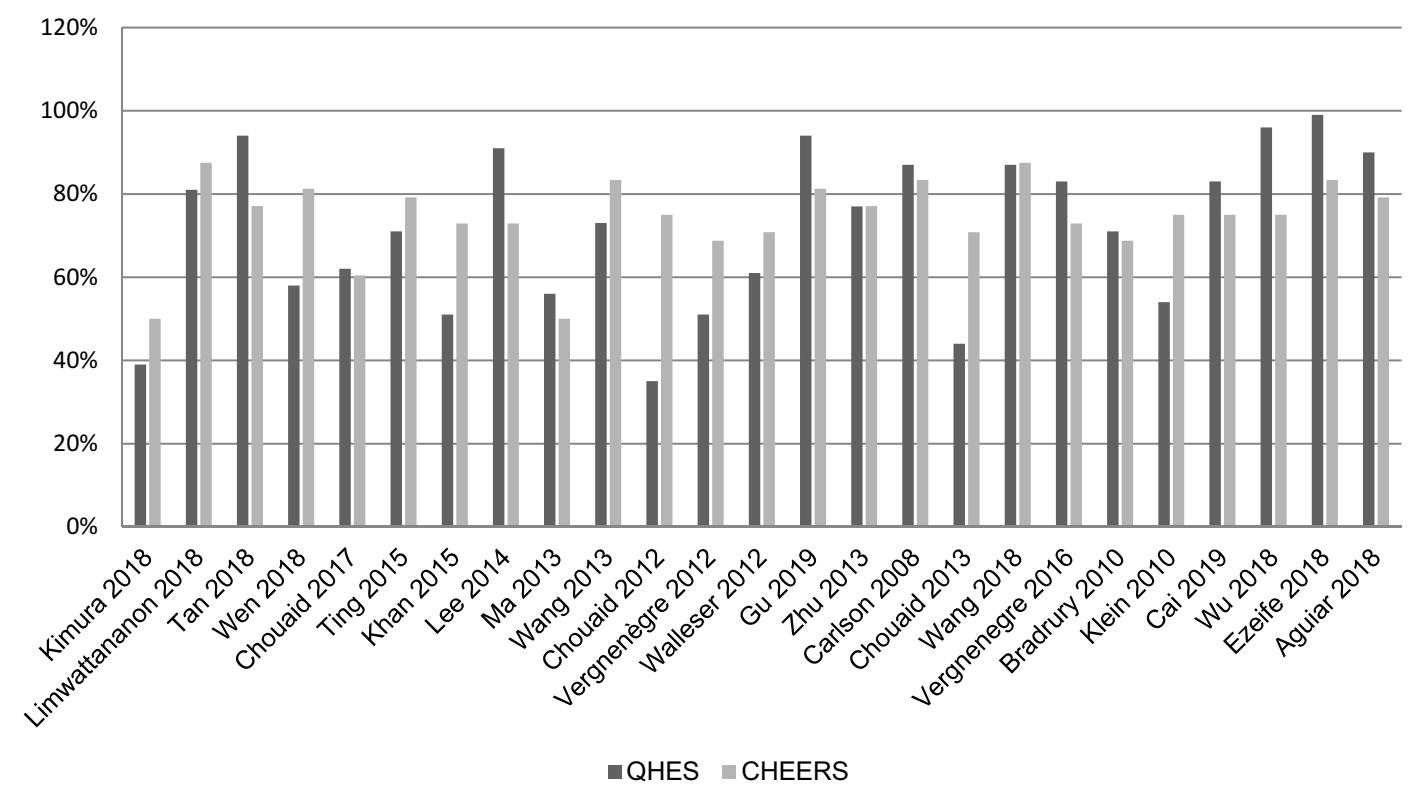

Figure 3 Comparison of qualitative CHEERS and QHES scores for each article examined. Statistical comparison (paired Wilcoxon rank test) of CHEERS with QHES scores did not result in a statistically significant difference between instruments (CHEERS vs QHES: $p=0.5 \mathrm{I}$ ). Scores for each article are illustrated in percentages to allow direct comparison. Light grey=CHEERS, dark grey=QHES.

denominator were not completely displayed in a clear, transparent manner.

\section{Discussion}

This review mainly analyzes the quality of articles published since 2000 on health economic evaluations (HEEs) of first-line targeted treatments (afatinib, gefitinib, erlotinib and osimertinib) used in the treatment of patients with metastatic NSCLC. Based on the results of CHEERS and QHES assessment, less than one in three studies are deemed to be of good quality. To support decision-making in health care it is important to ensure the reliability and consistency of pharmacoeconomic evaluation results. Among the different instruments that evaluate the quality of HEEs, the CHEERS checklist has been widely used since its publication in 2013, and more than 40 review articles in different medical fields have analyzed the adherence of economic evaluations with the CHEERS checklist.

In treating non-small-cell pulmonary carcinoma, about eight systematic reviews of HEEs have been performed previously, and three of them were conducted for quality assessment. Bongers et $\mathrm{al}^{38}$ evaluated the methodological quality of 11 full studies published between 2001 and 2010 using the Drummond checklist, consisting of an assessment of content structure and data identification, but they reported that there was no question included that addresses the inclusion of all relevant cost items. Lange et $\mathrm{al}^{39}$ and Nguyen et $\mathrm{al}^{40}$ used the
QHES instrument to evaluate the quality of the included studies. The scoring method of the two reviews is different: a three-level scale (zero score, half and full score) was modified by Nguyen et $\mathrm{al}^{40}{ }^{40}$ which only reviewed the economic evaluations of erlotinib in the first-line treatment, while there was no partial points awarded per item by Lang et $\mathrm{al}^{39}$ or for most other quality reviews.

According to Zhang, ${ }^{41}$ a quality review may serve two basic objectives: it may determine a minimum quality threshold or study design threshold, or be intended to interpret the differences in the results of selected studies, indicating the shortcomings and evaluating how to improve quality.

Both the CHEERS and the QHES checklists were utilized for examining the 25 studies included in the present analysis, and the quality review of the selected publications in this study revealed some common shortcomings.

According to the source of cost and utility data, how these data be used to model the natural course of disease progression remained unclear. The majority of the data were extracted from clinical trials with short durations, which may result in some observed differences from the actual longer term situation. There was only one study ${ }^{23}$ that collected resources monthly using case report forms (CRF), and followed up with participants until progression or death. Such a study with real-world data may be most appropriate for providing a model with disease progression. Furthermore, about one-third of the selected 
articles mentioned the simulation of transition probabilities, and four of them ${ }^{30,33,37,42}$ also demonstrated the calculation equation that was used. However, there was generally not enough explicit description and transparency included in the reviewed publications.

The general consistency in modeling for the same metastatic NSCLC reflects the results of major attempts in standardization of outcomes for research and clinical practice. Nevertheless, there remains room for improvement in the health economic evaluations of cancer-related interventions, including illustrating clearly the composition of metrics and transparently representing the source of parameters. The studies reviewed generally characterized uncertainty about the sampling and the effects on observed outcomes, but rarely discussed heterogeneity. As has already been indicated by many researchers, NSCLC may exhibit substantial heterogeneity, encompassing a spectrum of clinical and physiological manifestations, and this may also influence outcomes. Therefore, heterogeneous groups and their impact on reported differences in effects should be considered or explained. In addition, since there is a limited amount of RCT research for NSCLC, other data sources such as real-world monitoring and large-scale observational studies should be incorporated more broadly into economic evaluations.

Neither the CHEERS nor QHES consider the issue of threshold, which has been discussed frequently as a hot topic in recent years. As shown in Table 2, most studies stated the willingness to pay (WTP) threshold, and determinants of threshold were not specified in only six of the articles reviewed. There is no uniform criteria for threshold range in each country, except the NICE (National Institute for Health and Care Excellence) from the UK and the ICER (Institute for Clinical and Economic Review) from the US. Meanwhile, some problems still remain unresolved in these systems. The NICE typically recommends treatments for use in the NHS (National Health Service) with cost-effectiveness threshold ranges between $£ 20,000$ and $£ 30,000$, which has been criticized by some experts who have stated that "the threshold is indeed too high". However, Khan et $\mathrm{al}^{23}$ reported that erlotinib had about an $80 \%$ chance of being costeffective at thresholds between $£ 50,000$ and $£ 60,000$, twice as much as the reported NICE threshold. As for the ICER, this organization defined the US cost-effectiveness threshold between $\$ 50,000$ and $\$ 175,000$ per QALY gained. Although nearly all research papers set their WTP threshold under this range, it does not have the same legal effect in the USA as NICE recommendation does in the UK. Like Zhu et $\mathrm{al}^{28}$ and most Chinese scholars have cited three times the per capita gross domestic product (GDP) as a WTP threshold, and experience with the use of such GDP-based thresholds in national-level decision-making processes show them to lack country specificity and this, in addition to uncertainty in the modeled cost-effectiveness ratios, can lead to the wrong decisions being made on how to spend health-care resources. ${ }^{43}$ For these reasons, it can be considered to be feasible and probably desirable to operate a defined threshold range. An elasticity index may suitable for the supply side, and a WTP threshold from the societal perspective may be appropriate for the demand side estimates.

A declaration of funding sources and conflict of interests in the domain of health economics is important to avoid all doubt on biases, and this is evaluated by both CHEERS and QHES. ${ }^{40}$ Six studies included in the current review did not disclose their sources of funding, while almost all the studies included a conflict of interest declaration. This discrepancy may be explained by the presumption that a negative declaration on conflict of interest encompasses both topics. ${ }^{12}$ However, explicit statements should be made regarding this, to avoid and eliminate any doubts.

Most models identified by this review were trial-based, and replied on a limited number of older databases, that may not fully capture contemporary patient care, limiting the consideration of the true variation in the NSCLC progression patterns. Frameworks of future models should be informed based on clinical evidence, genotyping and data availability to ensure the validity of model results. In addition, further HEE research is warranted to employ patient-level models and to provide a better disposition of data sources to improve economic modeling accuracy in NSCLC.

This review study has several limitations and potentially confounding variables. First, the differences in scoring could be potentially related to the interpretation of the reviewers. For example, discriminating between studies being partially or fully reported was difficult, in some cases. Second, the CHEERS checklist score was accorded with an equal weight for each item, and one may question if every item is of the same importance, from the title to uncertainty analysis. The application of the QHES instrument is observer-dependent, which does not permit intermediate scores, ${ }^{44}$ and thus some important information will be lost in the practical utilization of this method. Consequently, it is somewhat arbitrary to carry out quality classifications according to Hong et $\mathrm{al}^{27}$ and Spiegel et al, ${ }^{31}$ for example. Third, it was decided to allocate a score of 0.5 for partial reporting when using the CHEERS 
checklist, and this may lead to an upgrade of the overall score of some selected studies; using a dichotomous rating akin to the QHES checklist would likewise have decreased the reporting quality of some. Finally, the validity of the model itself along with the adaptability of the results in the health economic environment should be considered.

\section{Conclusion}

In conclusion, this review found an increasing number of published cost-effectiveness analyses of targeted therapies in the treatment of NSCLC in the later years analyzed, eg, 2015-2019, as compared with the earlier years starting in 2000. However, the overall quality of the literature included is not high according to the CHEERS and QHES evaluations. The standardization and refinement of the model application, as well as the consideration and measurement of each parameter needs to be improved. Future models could be informed or justified based on two basic objectives: to ensure the validity of model results, and to improve economic modeling accuracy. Obviously, a reliable cost-effectiveness result is essential, and especially for its data sources, demographic heterogeneity, sensitivity analysis and threshold selection must be strongly considered. Evaluating the relevance, reliability and generalizability of these results are an indispensable support for valid decision-making on the allocation of scarce health-care resources.

\section{Acknowledgments}

We appreciate Dr. C. Benjamin Naman (Ningbo University China and University of California, San Diego - USA) for critical reading and linguistic editing of the manuscript.

\section{Author Contributions}

All authors contributed to data analysis, drafting or revising the article, gave final approval of the version to be published, and agree to be accountable for all aspects of the work.

\section{Disclosure}

This work was conducted without any specific grant from a funding agency in the public, commercial or non-profit sectors, and the authors have no conflicts of interest to disclose. The CHEERS and QHES checklists that were used required appropriate citation, while the systematic literature review was conducted using the PRISMA guidelines.

\section{References}

1. Xing PY, Zhu YX, Wang L, et al. What are the clinical symptoms and physical signs for non-small cell lung cancer before diagnosis is made? A nation-wide multicenter 10-year retrospective study in China. Cancer Med. 2019;8(8):4055-4069. doi:10.1002/cam4.2256

2. Meng H, Guo X, Sun D, et al. Genomic profiling of driver gene mutations in Chinese patients with non-small cell lung cancer. Front Genet. 2019;10:1008. doi:10.3389/fgene.2019.01008

3. Wu B, Gu XH, Zhang Q, et al. Cost-effectiveness of osimertinib in treating newly diagnosed, advanced EGFR-mutation-positive non-small cell lung cancer. Oncologist. 2019;24(3):349-357. doi:10.1634/theoncologist.2018-0150

4. Nonsquamous A, Lung NC, Klein R, et al. Cost-effectiveness of pemetrexed as first-line maintenance. Ann Oncol. 2010;5:1263-1272.

5. Aguiar PN, Haaland B, Park W, et al. Cost-effectiveness of osimertinib in the first-line treatment of patients with EGFR-mutated advanced non-small cell lung cancer. JAMA Oncol. 2018;8:1080-1084.

6. Chalkidou K, Marquez P, Dhillon PK, et al. Evidence-informed frameworks for cost-effective cancer care and prevention in low, middle, and high-income countries. Lancet Oncol. 2014;15(3):e119e131. doi:10.1016/S1470-2045(13)70547-3

7. Drummond MF, Jefferson TO. Guidelines for authors and peer reviewers of economic submissions to the BMJ. BMJ. 1996;313 (7052):275-283. doi:10.1136/bmj.313.7052.275

8. Drummond MF, Sculpher MJ, Torrance GW, et al. Methods for the Economic Evaluation of Health Care Programmes. 3rd. Oxford: Oxford University Press; 2005.

9. Evers S, Goossens M, Vet $\mathrm{H}$, et al. Criteria list for assessment of methodological quality of economic evaluations: consensus on health economic criteria. Int J Technol Assess Health Care. 2005;21 (2):240-245. doi:10.1017/S0266462305050324

10. Husereau D, Drummond M, Petrou S, et al. Consolidated health economic evaluation reporting standards (CHEERS) statement. BMJ. 2013;346(mar25 1):f1049. doi:10.1136/bmj.f1049

11. Motheral B. Assessing the value of the quality of health economic studies (QHES). J Manag Care Pharm. 2003;9(1):86-87. doi:10. 18553/jmcp.2003.9.1.86

12. Monten C, Veldeman L, Verhaeghe N, et al. A systematic review of health economic evaluation in adjuvant breast radiotherapy: quality counted by numbers. Radiother Oncol. 2017;125(2):186-192. doi:10.1016/j.radonc.2017.08.034

13. Ofman JJ, Sullivan SD, Neumann PJ, et al. Examining the value and quality of health economic analyses: implications of utilizing the QHES. J Manag Care Pharm. 2003;9(1):53-61. doi:10.18553/jmcp. 2003.9.1.53

14. Chiou CF, Hay JW, Wallace JF, et al. Development and validation of a grading system for the quality of cost-effectiveness studies. Med Care. 2003;41(1):32-44. doi:10.1097/00005650-200301000-00007

15. Klein R, Wielage R, Muehlenbein C, et al. Cost-effectiveness of pemetrexed as first-line maintenance therapy for advanced nonsquamous non-small cell lung cancer. $J$ Thorac Oncol. 2010;15 (8):1263-1272. doi:10.1097/JTO.0b013e3181e15d16

16. Ma Y, Huang Y, Zhao H, et al. The cost-effectiveness analysis of gefitinib or erlotinib in the treatment of advanced EGFR mutant non-small cell lung cancer patients. Zhongguo Fei Ai Za Zhi. 2013;16(4):203-210. doi:10.3779/j.issn.1009-3419.2013.04.06

17. Carlson JJ, Reyes C, Oestreicher N, Lubeck D, Ramsey SD, Veenstra DL. Comparative clinical and economic outcomes of treatments for refractory non-small cell lung cancer (NSCLC). Lung Cancer. 2008;61(3):405-415. doi:10.1016/j.lungcan.2007.12.023

18. Tan PT, Aziz MI, Pearce F, et al. Cost effectiveness analysis of afatinib versus pemetrexed-cisplatin for first-line treatment of locally advanced or metastatic EGFR mutation positive non-small-cell lung cancer from the Singapore healthcare payer's perspective. BMC Cancer. 2018;18(1):352. doi:10.1186/s12885-018-4223-y 
19. Chouaid C, Luciani L, LeLay K, et al. Cost-effectiveness analysis of afatinib versus gefitinib for first-line treatment of advanced EGFR-mutated advanced non-small cell lung cancers. J Thorac Oncol. 2017;12(10):1496-1502. doi:10.1016/j.jtho.2017.07.013

20. Vergnenegre A, Ray JA, Chouaid C, et al. Cross-market cost-effectiveness analysis of erlotinib as first-line maintenance treatment for patients with stable non-small cell lung cancer. Clinicoecon Outcomes Res. 2012;4:31-37. doi:10.2147/CEOR.S25923

21. Walleser S, Ray J, Bischoff $\mathrm{H}$, et al. Maintenance erlotinib in advanced nonsmall cell lung cancer: cost-effectiveness in EGFR wild-type across Europe. Clinicoecon Outcomes Res. 2012;4:269-275. doi:10.2147/CEOR.S31794

22. Kimura M, Yasue F, Usami E, et al. Cost-effectiveness and safety of the molecular targeted drugs afatinib, gefitinib and erlotinib as first-line treatments for patients with advanced EGFR mutation-positive non-small-cell lung cancer. Mol Clin Oncol. 2018;9(2):201-206. doi:10.3892/mco.2018.1640

23. Khan I, Morris S, Hackshaw A, et al. Cost-effectiveness of first-line erlotinib in patients with advanced non-small-cell lung cancer unsuitable for chemotherapy. BMJ Open. 2015;5(7):e006733. doi:10.1136/ bmjopen-2014-006733

24. Christos C, Herve LC, Chrystelle L, et al. Cost effectiveness of erlotinib versus chemotherapy for first-line treatment of non small cell lung cancer (NSCLC) in fit elderly patients participating in a prospective Phase 2 study (GFPC 0504). BMC Cancer. 2012;12 (1). doi:10.1186/1471-2407-12-301.

25. Chouaid C, Caer HL, Corre R, et al. Cost analysis of erlotinib versus chemotherapy for first-line treatment of non-small-cell lung cancer in frail elderly patients participating in a prospective phase 2 study (GFPC 0505). Clin Lung Cancer. 2013;14(2):103-107. doi:10.1016/ j.cllc.2012.04.006

26. Bradbury PA, Tu D, Seymour L, et al. Analysis, economic analysis: randomized placebo-controlled clinical trial of erlotinib in advanced non-small cell lung cancer. J Natl Cancer Inst. 2010;102(5):298-306. doi:10.1093/jnci/djp518

27. Hong D, Si L, Jiang M, et al. Cost effectiveness of sodium-glucose cotransporter-2 (SGLT2) inhibitors, glucagon-like peptide-1 (GLP-1) receptor agonists, and dipeptidyl peptidase-4 (DPP-4) inhibitors: a systematic review. Pharmacoeconomics. 2019;37(12):777-818. doi:10.1007/s40273-019-00833-1

28. Zhu J, Li T, Wang X, et al. Gene-guided gefitinib switch maintenance therapy for patients with advanced EGFR mutation-positive non-small cell lung cancer: an economic analysis. BMC Cancer. 2013;13(1):39. doi:10.1186/1471-2407-13-39

29. Vergnenegre A, Massuti B, Marinis F, et al. Economic analysis of first-line treatment with erlotinib in an EGFR-mutated population with advanced NSCLC. J Thorac Oncol. 2016;11(6):801-807. doi:10.1016/j.jtho.2016.02.004

30. Gu X, Zhang Q, Chu YB, et al. Cost-effectiveness of afatinib, gefitinib, erlotinib and pemetrexed-based chemotherapy as first-line treatments for advanced non-small cell lung cancer in China. Lung Cancer. 2019;127:84-89. doi:10.1016/j.lungcan.2018.11.029

31. Spiegel BMR, Targownik LE, Kanwal F, et al. The quality of published health economic analyses in digestive diseases: a systematic review and quantitative appraisal. Gastroenterology. 2004;127 (2):403-411. doi:10.1053/j.gastro.2004.04.020
32. Limwattananon C, Limwattananon S, Waleekhachonloet $\mathrm{O}$, et al. Cost-effectiveness analysis of policy options on first-line treatments for advanced, non-small cell lung cancer in Thailand. Lung Cancer. 2018;120:91-97. doi:10.1016/j.lungcan.2018.04.003

33. Wen $\mathrm{F}$, Zheng $\mathrm{H}$, Zhang $\mathrm{P}$, et al. OPTIMAL and ENSURE trials-based combined cost-effectiveness analysis of erlotinib versus chemotherapy for the first-line treatment of Asian patients with non-squamous non-small-cell lung cancer. BMJ Open. 2018;8(4): e020128. doi:10.1136/bmjopen-2017-020128

34. Lee VW, Schwander B, Lee VH. Effectiveness and cost-effectiveness of erlotinib versus gefitinib in first-line treatment of epidermal growth factor receptor-activating mutation-positive non-small-cell lung cancer patients in Hong Kong. Hong Kong Med J. 2014;20(3):178-186. doi: $10.12809 / \mathrm{hkmj} 133986$

35. Ting J, Tien Ho P, Xiang $\mathrm{P}$, et al. Cost-effectiveness and value of information of erlotinib, afatinib, and cisplatin-pemetrexed for first-line treatment of advanced EGFR mutation-positive non-smallcell lung cancer in the United States. Value Health. 2015;18 (6):774-782. doi:10.1016/j.jval.2015.04.008

36. Wang S, Peng L, Li J, et al. A trial-based cost-effectiveness analysis of erlotinib alone versus platinum-based doublet chemotherapy as first-line therapy for Eastern Asian nonsquamous non-small-cell lung cancer. PLoS One. 2013;8(3):e55917. doi:10.1371/journal. pone.0055917

37. Wang H, Zeng C, Li X, et al. Cost-utility of afatinib and gefitinib as first-line treatment for EGFR-mutated advanced non-small-cell lung cancer. Future Oncol. 2019;15(2):181-191. doi:10.2217/fon-2018-0692

38. Bongers ML, Coupe VM, Jansma EP, et al. Cost effectiveness of treatment with new agents in advanced non-small-cell lung cancer a systematic review. Pharmacoeconomics. 2012;30(1):17-34. doi:10.2165/11595000-000000000-00000

39. Lange A, Prenzler A, Frank M, et al. A systematic review of the cost-effectiveness of targeted therapies for metastatic non-small cell lung cancer (NSCLC). BMC Pulm Med. 2014;14(1):192. doi:10.1186/1471-2466-14-192

40. Nguyen TK, Goodman CD, Boldt RG, et al. Evaluation of health economics in radiation oncology: a systematic review. Int $J$ Radiat Oncol Biol Phys. 2016;94(5):1006-1014. doi:10.1016/j. ijrobp.2015.12.359

41. Zhang TS, Zhong WZ, Li B. Applied Methodology for EvidenceBased Medicine. 2nd ed. Changsha: CSUPRESS; 2015.

42. Cai HF, Zhang LF, Li N, et al. Cost-effectiveness of osimertinib as first-line treatment and sequential therapy for EGFR mutation-positive non-small cell lung cancer in China. Clin Ther. 2019;41(2):280-290. doi:10.1016/j.clinthera.2018.12.007

43. Bertram MY, Lauer JA, De Joncheere K, et al. Cost-effectiveness thresholds: pros and cons. Bull World Health Organ. 2016;94 (12):925-930. doi:10.2471/BLT.15.164418

44. Gerkens S, Crott R, Cleemput I, et al. Comparison of three instruments assessing the quality of economic evaluations: a practical exercise on economic evaluations of the surgical treatment of obesity. Int J Technol Assess Health Care. 2008;24(03):318-325. doi:10.1017/S0266462308080422

45. Ezeife DA, Kirk V, Chew DS, et al. Economic analysis of osimertinib in previously untreated EGFR-mutant advanced non-small cell lung cancer in Canada. Lung Cancer. 2018;125:1-7. doi:10.1016/j. lungcan.2018.08.024 


\section{Publish your work in this journal}

Cancer Management and Research is an international, peer-reviewed open access journal focusing on cancer research and the optimal use of preventative and integrated treatment interventions to achieve improved outcomes, enhanced survival and quality of life for the cancer patient.
The manuscript management system is completely online and includes a very quick and fair peer-review system, which is all easy to use. Visit http://www.dovepress.com/testimonials.php to read real quotes from published authors.

Submit your manuscript here: https://www.dovepress.com/cancer-management-and-research-journal 